\title{
Scattering of a Laser Beam on an Ensemble of Asymmetrical Erythrocytes
}

\author{
S. Yu. Nikitin ${ }^{a}$, V. D. Ustinov ${ }^{b}$, E. G. Tsybrov ${ }^{c, *}$, and M. S. Lebedeva ${ }^{a}$ \\ ${ }^{a}$ Faculty of Physics, Moscow State University, Moscow, 119991 Russia \\ ${ }^{b}$ Center of Fundamental and Applied Mathematics, Moscow State University, Moscow, 119991 Russia \\ ${ }^{c}$ Faculty of Computational Mathematics and Cybernetics, Moscow State University, Moscow, 119991 Russia \\ *e-mail: tsybrovevgeniy@yandex.ru \\ Received October 27, 2020; revised December 27, 2020; accepted February 22, 2021
}

\begin{abstract}
The study describes an analytical model of laser beam scattering on an inhomogeneous ensemble of erythrocytes. The model takes into account erythrocyte variation in size, shape, and spatial orientation. A relationship is established between an experimentally determined parameter: visibility of the diffraction pattern, and a characteristic serving as a measure of inhomogeneity of erythrocyte size and shape in a blood specimen. This relationship is shown to be monotonous, which means that erythrocyte variation in size and shape can be assessed based on measurements of the diffraction pattern visibility.
\end{abstract}

Keywords: diffraction pattern, erythrocyte, visibility, size distribution

DOI: $10.1134 / \mathrm{S} 0030400 \mathrm{X} 21070122$

\section{INTRODUCTION}

Laser diffraction analysis of a blood smear can be considered a method of assessing the size distribution of erythrocytes (red blood cells, RBC) [1]. These measurements are performed by illuminating a blood smear with a laser beam, recording the produced diffraction pattern, and analyzing it using a specialized computer algorithm. The advantages of this method are its relative simplicity and the possibility of rapidly analyzing large blood cell ensembles without using microscopy. The algorithm of diffraction pattern analysis is based on solving the physical problem of laser beam scattering on an inhomogeneous ensemble of particles. This problem includes a certain model of an $\mathrm{RBC}$ ensemble. In our previous study [2], erythrocytes in a blood smear were modeled by round discs varying in radius. A more accurate model should also take into account the specific features of RBC shape. In the present work, an RBC ensemble in a blood smear is modeled with a set of flat elliptical discs varying in size, shape, and orientation. Our principal goal was to establish a relationship between the statistical characteristics of this ensemble and the parameters of the observed diffraction pattern.

From the medical point of view, it is necessary to mention that an important blood test parameter is $\mathrm{RBC}$ distribution width (RDW). It is known that an increased value of this parameter (higher than $15 \%$ ) is a nonspecific indicator of general poor health condition [3]. Elevated RDW values are considered an unfavorable marker in patients with cardiovascular disor- ders, liver and kidney diseases [4], as well as with severe acute pneumonia caused by coronavirus infection (COVID-19) [5].

\section{LASER DIFFRACTION ANALYSIS AS A METHOD OF PARTICLE SIZE MEASUREMENT}

Applications of laser beam diffraction for size measurement in small particles have been developing since the 1980s [6]. Currently, this technique is utilized for quality control of powders composed of small particles, such as medical and chemical preparations, biological cells, bottom silt suspensions, or paint microspheres [7]. However, in most studies using laser diffraction, particles are modeled with spheres or thin round discs, which implies an error in the representation of real nonspherical particles [5]. This does not constitute a fundamental obstacle for applying the method, since it still produces consistent results in similar samples of small particles and different results in significantly different ones. Nevertheless, for certain applications, such as medical blood testing, it is critically important that the measurements results be represented adequately [8]. For this purpose, more accurate models of laser beam scattering on inhomogeneous ensembles of particles are required [9]. One of such models is proposed in the present study.

In medical practice, RBC size measurements are most commonly performed using a Coulter counter and standard optical microscopy [6]. Both these 
approaches have their advantages and shortcomings; here, it should be mentioned that evaluating the size of a higher number of erythrocytes requires a proportional increase in the duration of measurement. In contrast, to increase the number of erythrocytes accessed by laser diffraction analysis, it suffices to use a wider laser beam. In particular, dozens of thousands to million of erythrocytes can be studied in a single act of measurement that lasts for approximately a second, including computer data analysis. Importantly, the essential preparation of the blood smear specimen prior to the measurement may be significantly more time-consuming. Nevertheless, the possibility of rapid analysis of large RBC ensembles is a fundamental advantage of laser diffraction analysis in comparison to the competing techniques. Furthermore, the data of laser diffraction analysis can complement and clarify the data obtained using a Coulter counter and optical microscopy.

It was relatively recently that laser diffraction measurements have been introduced for RBC tests [1013]. A number of studies have addressed the direct problem of scattering of a plane monochromatic wave on a single nonspherical particle simulating an erythrocyte [14-17]. In the direct problem, all parameters of the laser beam and characteristics of the particle are known, and it is required to calculate the diffraction pattern.

The solution of the direct problem is usually employed as a ready component for solving the inverse problem where it is required to find the distribution function of particle sizes based on the given diffraction pattern. The inverse problem assumes that the particles in question are exposed to a laser beam of a known wavelength, and the diffraction pattern is recorded so as to obtain the angular distribution of the intensity of light scattered on these particles. Diffraction is measured in the far field and at small angles (up to $15^{\circ}-25^{\circ}$ deviation from the beam direction, depending on the problem). A number of studies have addressed this problem in the general form (for instance, see [7] for review).

There exist commercially available devices for laser diffraction analysis known as laser particle sizers [18]. Although the manufacturers do not disclose certain details of their design, on the whole, they are all based on the same principle. The inverse problem amounts to solving the following Fredholm integral equation of the first kind [7]:

$$
\int_{r_{1}}^{r_{2}} \tilde{I}(r, \theta) w(r) d r=I(\theta),
$$

where $w(r)$ is the particle size distribution sought for. Function $\tilde{I}(r, \theta)$ describes the angular distribution of the intensity of light scattered on a single round or spherical particle of radius $r$. Function $I(\theta)$ is the angular distribution of the intensity of light scattered on the entire ensemble of particles. This function is determined experimentally. This problem is considered to be ill-posed in the sense that its solution is unstable and strongly depends on noise [19]. It can be solved using various regularization techniques [20,21].

By replacing the integral in this equation with an integral sum with a finite number of terms and introducing a finite set of observation angles $\theta_{j}$, we obtain a system of linear algebraic equations:

$$
\sum_{i=1}^{N} \tilde{I}\left(r_{i}, \theta_{j}\right) p_{i}=I_{j}, \quad 1 \leq j \leq M,
$$

where $p_{i}=p\left(r_{i}\right)$ is the probability that the radius of an erythrocyte is $r_{i}$, and $I_{j}=I\left(\theta_{j}\right)$ is the intensity of light scattered at the angle $\theta_{j}$. By solving this system of equations, it is basically possible to find the entire distribution function of RBC sizes, but this requires measuring the distribution of light intensity in a broad field of the diffraction pattern. However, if the measurements of intensity are limited to two angles (in the first dark and light rings of the diffraction pattern), it is possible to find the first two moments of the distribution function of RBC sizes [2, 22].

A typical micrograph of a blood smear is shown in Fig. 1a. Figure $1 \mathrm{~b}$ shows a typical diffraction pattern produced by scattering of a $650-\mathrm{nm}$ laser beam on a wet smear. This diffraction pattern always features a characteristic round light spot in the center surrounded by a dark ring at some distance from the center, then by a light ring, and further by intermittent dark and light rings. On the whole, the structure resembles the well-known Airy disc pattern produced by diffraction of a plane wave incident on a circular aperture [23]. Visibility $v$ of the diffraction pattern is defined by the following formula [2]:

$$
v=\frac{I_{\max }-I_{\min }}{I_{\max }+I_{\min }}
$$

where $I_{\min }$ is the intensity of light in the first minimum of the diffraction pattern and $I_{\max }$ is the intensity in the subsequent maximum of the diffraction pattern. Visibility of the diffraction pattern can be employed to estimate the dispersion of particle sizes [2].

In our previous works [1, 2, 22], erythrocytes were modeled with round discs. In the present study, we generalize the theory by considering particles of more complex shapes. First, we will present the theoretical model describing the scattering of a laser beam on particles of elliptical shape with random orientation in the plane of the microscopic slide. Next, we will introduce a measure of asymmetry of arbitrarily shaped particles. Finally, we will describe numerical calculations undertaken to test the theory. From the mathematical point of view, our approach is close to the approach employed in [24-26]; however, it is different in that it is sensitive to the shape of erythrocytes in a blood 
(a)

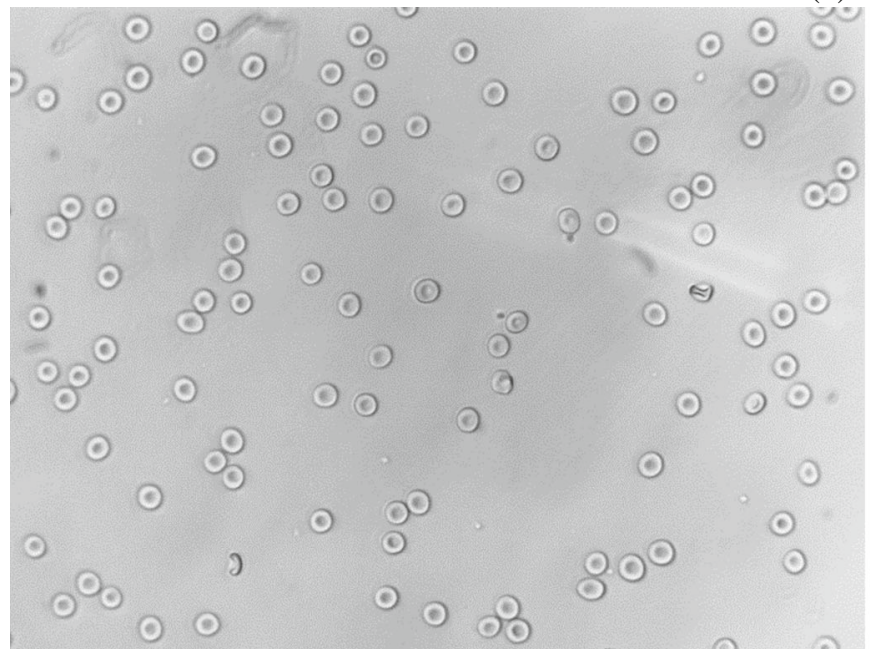

(b)

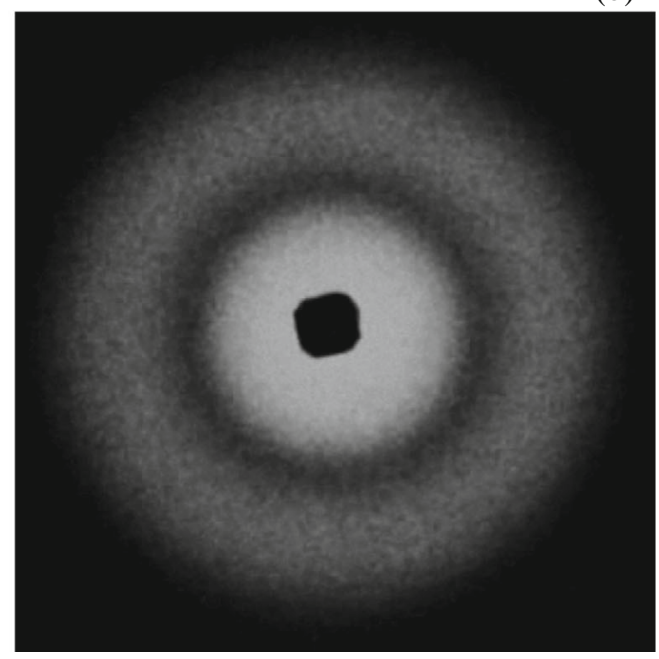

Fig. 1. Micrograph of a blood smear (a) and the pattern on laser beam scattering on a blood smear (b).

smear. This analysis can be used to estimate the share of sickle cells, echinocytes, poikilocytes, and other irregularly shaped cells that may for various reasons be present in the blood specimen tested.

\section{SCATTERING OF A LASER BEAM ON AN ENSEMBLE OF ELLIPTICAL DISCS}

Figure 1 shows the microscopic view of a blood smear and the pattern of laser beam scattering on the smear (diffraction pattern). It can be seen that erythrocytes vary in size and shape. They are not ideally round discs. Erythrocyte orientation in the smear can be determined by a straight line connecting the two most remote points of the cell. For an elliptical erythrocyte, this line is directed along the major axis of the ellipse. We assume this orientation to be arbitrary. A typical diffraction pattern exhibits circular symmetry and represents a system of concentric dark and light rings. It is known that the radius of the first dark ring is determined by the average RBC size in the smear, and the ratio between the brightness of the first dark and the first light ring is related to the dispersion of particle sizes [2]. It is unclear how the diffraction pattern is affected by variation of RBC shapes. This problem will be analyzed in the present work. Its main focus will be on calculating visibility of the diffraction pattern arising as a result of laser beam scattering on an ensemble of particles that exhibit variation in both size and shape.

An erythrocyte in a blood smear will be modeled with a flat elliptical disc. Scattering of a laser beam on this disc is described by the following formula [2]:

$$
I=I_{0}|\gamma|^{2}\left(a b \frac{k}{z}\right)^{2}\left[\frac{J_{1}(q)}{q}\right]^{2} .
$$

Here $I_{0}$ is the intensity of the incident beam, $|\gamma|^{2}$ is the parameter depending on the disc thickness and its optical density, $a$ and $b$ are the lengths of the disc semiaxes, $k$ is the wave number of the light wave, $z$ is the distance from the smear to the observation screen, and $J_{1}(q)$ is the Bessel function of the first order. The argument of this function is

$$
q=\frac{k}{z} \sqrt{a^{2} x^{2}+b^{2} y^{2}}
$$

where $x, y$ are the Cartesian coordinates of the point on the observation screen. Formulas (2) and (3) describe the distribution of light intensity in the part of the observation screen that lies outside of the direct laser beam incidence. The coordinate axes on the observation screen are parallel to the symmetry axes of the disc.

The intensity of light in the center of the diffraction pattern can be found using (2) and (3), setting $x \rightarrow 0$ and $y \rightarrow 0$. Taking into account the asymptotic behavior of the Bessel function [27], we obtain

$$
I(0)=\frac{1}{4} I_{0}|\gamma|^{2}\left(a b \frac{k}{z}\right)^{2} .
$$

The value $I(0)$ can serve as a convenient scale of light intensity in the diffraction pattern. The intensity of light at an arbitrary point of the diffraction pattern normalized to the intensity of the central diffraction maximum is given by

$$
\frac{I}{I(0)}=\left[\frac{2 J_{1}(q)}{q}\right]^{2} .
$$

Here, the value $q$ is given by formula (3). 
In vicinity of the first minimum of light intensity in the diffraction pattern, the Bessel function can be approximated by a linear function [27]:

$$
J_{1}(q)=\beta\left(q-q_{1}\right) .
$$

Here

$$
\beta=-0.4, \quad q_{1}=3.82 .
$$

In this zone, the distribution of normalized light intensity has the form

$$
\frac{1}{4 \beta^{2}} \frac{I}{I(0)}=\left(1-\frac{q_{1}}{q}\right)^{2} .
$$

Suppose that the elliptical disc has small eccentricity (i.e., its deviation from a round disc is small). In this case, the lengths of the semiaxes can be represented as follows:

$$
a=a_{0}\left(1+\varepsilon_{2}\right), \quad b=a_{0}\left(1-\varepsilon_{2}\right) .
$$

Here $\varepsilon_{2}$ is a small dimensionless parameter, such that

$$
\left|\varepsilon_{2}\right| \ll 1 \text {. }
$$

By substituting (7) into (3) and taking (8) into account, we obtain the following approximation for $q$ :

$$
q=\frac{k}{z} a_{0} \sqrt{x^{2}+y^{2}}\left(1+\varepsilon_{2} \frac{x^{2}-y^{2}}{x^{2}+y^{2}}\right) .
$$

Formulas (5), (6), and (9) describe the scattering of a laser beam on an elliptical disc with low eccentricity.

Let us now suppose that the symmetry axes of the disc are rotated by an angle $\varphi$ relative to the Cartesian axes on the observation screen. Then the coordinates $x, y$ in (9) should be substituted with novel coordinates according to the following rule

$$
x \rightarrow x \cos \varphi+y \sin \varphi, \quad y \rightarrow-x \sin \varphi+y \cos \varphi .
$$

The expression for $q$ acquires the following form:

$q=\frac{k}{z} a_{0} \sqrt{x^{2}+y^{2}}\left[1+\varepsilon_{2} \frac{\left(x^{2}-y^{2}\right) \cos 2 \varphi+2 x y \sin 2 \varphi}{x^{2}+y^{2}}\right]$,

or

$$
q=\sqrt{g}\left[1+\varepsilon_{2} \frac{h \cos 2 \varphi+2 u v \sin 2 \varphi}{g}\right],
$$

where

$$
u=\frac{k a_{0}}{z} x, \quad v=\frac{k a_{0}}{z} y
$$

and

$$
g=u^{2}+v^{2}, \quad h=u^{2}-v^{2} .
$$

From (8) and (11), it follows that the value $q_{1} / q$ can be approximated by:

$$
\frac{q_{1}}{q}=\frac{q_{1}}{\sqrt{g}}-\varepsilon_{2} \frac{q_{1}}{\sqrt{g}} \frac{h \cos 2 \varphi+2 u v \sin 2 \varphi}{g} .
$$

By substituting (14) into (6), we obtain

$$
\begin{gathered}
\frac{1}{4 \beta^{2}} \frac{I}{I(0)}=\left(1-\frac{q_{1}}{\sqrt{g}}\right)^{2} \\
+2 \varepsilon_{2} \frac{q_{1}}{\sqrt{g}}\left(1-\frac{q_{1}}{\sqrt{g}}\right) \frac{h \cos 2 \varphi+2 u v \sin 2 \varphi}{g} \\
+\left[\varepsilon_{2} \frac{q_{1}}{\sqrt{g}} \frac{h \cos 2 \varphi+2 u v \sin 2 \varphi}{g}\right]^{2} .
\end{gathered}
$$

Formula (15) describes the scattering of a laser beam on an arbitrarily oriented elliptical disc with small eccentricity.

Next, suppose that the rotation angle between the elliptical disc and the Cartesian axes is a random variable with a probability density distribution $w(\varphi)=\frac{1}{2 \pi}$. This means that all orientations of the discs are equiprobable. By averaging (15) over the angle $\varphi$, we obtain:

$$
\frac{1}{4 \beta^{2}} \frac{\langle I\rangle_{\varphi}}{I(0)}=\left(1-\frac{q_{1}}{\sqrt{g}}\right)^{2}+\frac{1}{2} \varepsilon_{2}^{2}\left(\frac{q_{1}}{\sqrt{g}}\right)^{2} .
$$

Equation (16) was derived taking into account (13), as well as the fact that

$$
\begin{gathered}
\langle\cos 2 \varphi\rangle_{\varphi}=\langle\sin 2 \varphi\rangle_{\varphi}=\langle\sin 4 \varphi\rangle_{\varphi}=0, \\
\left\langle\cos ^{2} 2 \varphi\right\rangle_{\varphi}=\left\langle\sin ^{2} 2 \varphi\right\rangle_{\varphi}=\frac{1}{2} .
\end{gathered}
$$

Thus, formula (16) describes the scattering of a laser beam on chaotically oriented elliptical discs with small eccentricity.

Next, suppose that the parameter $\varepsilon_{2}$, which describes the disc eccentricity, is a random variable with the following characteristics:

$$
\left|\varepsilon_{2}\right| \ll 1, \quad\left\langle\varepsilon_{2}\right\rangle=0, \quad\left\langle\varepsilon_{2}^{2}\right\rangle=\sigma_{2}^{2} .
$$

Then, by averaging expression (16) over $\varepsilon_{2}$, we obtain

$$
\frac{1}{4 \beta^{2}} \frac{\langle I\rangle_{\varphi, \varepsilon}}{I(0)}=\left(1-\frac{q_{1}}{\sqrt{g}}\right)^{2}+\frac{1}{2} \sigma_{2}^{2}\left(\frac{q_{1}}{\sqrt{g}}\right)^{2} .
$$

Formula (18) describes the scattering of a laser beam on chaotically oriented elliptical discs with random eccentricity. In this formula, parameter $g$ is given by (12) and (13). Using these formulas, we obtain

$$
\sqrt{g}=\frac{k a_{0}}{z} \sqrt{x^{2}+y^{2}}
$$

Here $a_{0}$ is the parameter describing the size of the elliptical disc.

Now assume that the parameter $a_{0}$, which describes the size of the elliptical disc, is also a random variable. We will define it by the formula

$$
a_{0}=R_{0}\left(1+\varepsilon_{1}\right) \text {. }
$$


Here, the value $R_{0}$ describes the mean size of the elliptical discs, and the parameter $\varepsilon_{1}$ is a random variable with the following characteristics:

$$
\left|\varepsilon_{1}\right| \ll 1, \quad\left\langle\varepsilon_{1}\right\rangle=0, \quad\left\langle\varepsilon_{1}^{2}\right\rangle=\sigma_{1}^{2} .
$$

Formulas (21) imply that inhomogeneity of the ensemble of elliptical discs that model erythrocytes in a blood smear is relatively low. Dispersion (variability) of RBC size is characterized by the value $\sigma_{1}^{2}$.

By substituting (20) into (19), we obtain

$$
\sqrt{g}=\left(1+\varepsilon_{1}\right) \frac{k R_{0}}{z} \sqrt{x^{2}+y^{2}} .
$$

Hence

$$
\frac{q_{1}}{\sqrt{g}}=\frac{q_{1}}{\left(1+\varepsilon_{1}\right) \frac{k R_{0}}{z} \sqrt{x^{2}+y^{2}}}
$$

or

$$
\frac{q_{1}}{\sqrt{g}}=\frac{P_{0}}{1+\varepsilon_{1}}
$$

where

$$
P_{0}=\frac{q_{1} z}{k R_{0} \sqrt{x^{2}+y^{2}}} .
$$

By substituting (22) into (18), we obtain

$$
\frac{1}{4 \beta^{2}} \frac{\langle I\rangle_{\varphi, \varepsilon}}{I(0)}=\left(1-\frac{P_{0}}{1+\varepsilon_{1}}\right)^{2}+\frac{1}{2} \sigma_{2}^{2}\left(\frac{P_{0}}{1+\varepsilon_{1}}\right)^{2} .
$$

Taking into account (21), it can be approximated by

$$
\frac{1}{4 \beta^{2}} \frac{\langle I\rangle_{\varphi, \varepsilon}}{I(0)}=\left(1-P_{0}+P_{0} \varepsilon_{1}\right)^{2}+\frac{1}{2} \sigma_{2}^{2} P_{0}^{2}\left(1-2 \varepsilon_{1}+\varepsilon_{1}^{2}\right)
$$

or

$$
\begin{aligned}
& \frac{1}{4 \beta^{2}} \frac{\langle I\rangle_{\varphi, \varepsilon}}{I(0)}=\left(1-P_{0}\right)^{2}+2 \varepsilon_{1} P_{0}\left(1-P_{0}\right) \\
& +\left(P_{0} \varepsilon_{1}\right)^{2}+\frac{1}{2} \sigma_{2}^{2} P_{0}^{2}\left(1-2 \varepsilon_{1}+\varepsilon_{1}^{2}\right)
\end{aligned}
$$

By averaging this expression over $\varepsilon_{1}$, we obtain

$$
\frac{1}{4 \beta^{2}} \frac{\langle I\rangle_{\varphi, \varepsilon, \varepsilon_{1}}}{I(0)}=\left(1-P_{0}\right)^{2}+\sigma_{1}^{2} P_{0}^{2}+\frac{1}{2} \sigma_{2}^{2} P_{0}^{2}\left(1+\mu_{1}\right)
$$

or approximately

$$
\frac{1}{4 \beta^{2}} \frac{\langle I\rangle_{\varphi, \varepsilon, \varepsilon_{1}}}{I(0)}=\left(1-P_{0}\right)^{2}+\left(\sigma_{1}^{2}+\frac{1}{2} \sigma_{2}^{2}\right) P_{0}^{2} .
$$

Formula (23) describes the distribution of normalized intensity of light in vicinity of the first dark ring of the diffraction pattern produced by scattering of a laser beam on a blood smear. It takes into account RBC variation in size, shape, and spatial orientation. In this formula, $\langle I\rangle_{\varphi, \varepsilon, \varepsilon_{1}}$ is the intensity of light at a given point of the observation screen, $\beta=-0.4$ and $q_{1}=3.82$ are constant values (Bessel function parameters), $I(0)$ is the intensity of light in the center of the diffraction pattern, $\sigma_{2}^{2}$ is the measure of RBC variation in shape, and $\sigma_{1}^{2}$ is the measure of RBC variation in size. Parameter $P_{0}$ is given by the formula

$$
P_{0}=\frac{q_{1} z}{k R_{0} \sqrt{x^{2}+y^{2}}},
$$

where $x, y$ are the coordinates of the point on the observation screen, $R_{0}$ is the characteristic of the mean $\mathrm{RBC}$ size, $k$ is the wave number of the light wave, and $z$ is the distance from the blood smear to the observation screen.

As it can be seen from (23), normalized intensity of light in the first minimum of the diffraction pattern is achieved under condition of

$$
P_{0}=1
$$

and is given by

$$
\frac{1}{4 \beta^{2}} \frac{\langle I\rangle_{\min }}{I(0)}=\sigma_{1}^{2}+\frac{1}{2} \sigma_{2}^{2}
$$

A diffraction pattern parameter that can be assessed experimentally is the pattern visibility in vicinity of the first minimum of light intensity (the first dark ring). We will define it by formula (1). If only the size inhomogeneity of the particle ensemble is taken into account, the visibility of the diffraction pattern in vicinity of the first minimum of light intensity is approximately expressed by the following formula [2]:

$$
V=1-76 \sigma_{1}^{2} \text {. }
$$

This formula is true in the limiting case of weak inhomogeneity of the ensemble when

$$
\sigma_{1}^{2}=\left\langle\varepsilon_{1}^{2}\right\rangle=\frac{\left\langle(R-\langle R\rangle)^{2}\right\rangle}{\langle R\rangle^{2}} \equiv \frac{\sigma_{R}^{2}}{\langle R\rangle^{2}} \ll 1 .
$$

If we take into account inhomogeneity of the RBC ensemble in both size and shape, the formula for visibility of the diffraction pattern takes the form

$$
V=1-76 \sigma^{2}
$$

Here

$$
\sigma^{2}=\sigma_{1}^{2}+\frac{1}{2} \sigma_{2}^{2}
$$

$\sigma_{1}^{2}$ is the measure of RBC variation in size, $\sigma_{2}^{2}$ is the measure of RBC variation in shape, and $\sigma^{2}$ is a com- 
bined parameter describing $\mathrm{RBC}$ variation in both size and shape. From (28), it follows that

$$
\sigma=\sqrt{\frac{1-v}{76}}
$$

This formula represents the principal result of our computation. It establishes a relationship between the visibility $v$ of the diffraction pattern in vicinity of the first minimum of light intensity and the parameter $\sigma$ describing size and shape inhomogeneity of the RBC ensemble in the blood sample studied. Formula (30) can be applied to weakly inhomogeneous RBC ensembles. Thus, by measuring visibility of the diffraction pattern produced by laser beam scattering on a blood smear and using formula (30), it is possible to estimate the variation of RBC size and shape in the blood sample tested.

\section{MEASURE OF ERYTHROCYTE ASYMMETRY}

In this section, we introduce a measure of $\mathrm{RBC}$ asymmetry in a blood smear without making any specific assumptions about the cell shape. This will provide a possibility to assess quantitatively the asymmetry of real erythrocytes in a blood smear. This measure can be introduced by comparing the shapes of an erythrocyte and a circle of equal area. Let us define this measure as follows:

$$
M=\frac{\Delta S}{S}=\frac{S-S_{\mathrm{com}}}{S},
$$

where $S_{\text {com }}$ is the area of the common part of an erythrocyte in the blood smear and an equal-sized circle, and $S$ is the area of the circle equal to that of the erythrocyte. It is assumed that the circle and the figure representing the erythrocyte are positioned in such a way that the area of their common part is maximal. In particular, the measure of asymmetry of a round erythrocyte is equal to zero.

Let us apply this definition to a figure of elliptical shape and find out the relationship between the measure of asymmetry $M$, as defined by (31), and the ellipse asymmetry parameter $\varepsilon_{2}$ given by (7). It can be shown (see Supplement) that this relationship is expressed by the following formula:

$$
\varepsilon_{2}=\frac{\pi}{2} M
$$

From (32), it follows that

$$
\sigma_{2}^{2}=\left\langle\varepsilon_{2}^{2}\right\rangle=\frac{\pi^{2}}{4}\left\langle M^{2}\right\rangle .
$$

Here, the angular brackets denote averaging over an RBC ensemble. By substituting (33) into (29), we obtain

$$
\sigma^{2}=\sigma_{1}^{2}+\frac{\pi^{2}}{8}\left\langle M^{2}\right\rangle
$$

Formulas (28) and (34) can be applied to weakly inhomogeneous RBC ensembles. In these formulas, the parameters $v, \sigma_{1}^{2}$, and $M$ are given by (1), (27), and (31). The parameters $\sigma_{1}^{2}$ and $\left\langle M^{2}\right\rangle$ can be explicitly written as follows:

$$
\sigma_{1}^{2}=\frac{\left\langle(R-\langle R\rangle)^{2}\right\rangle}{\langle R\rangle^{2}}, \quad\left\langle M^{2}\right\rangle=\left\langle\left(1-\frac{S_{\text {com }}}{S}\right)^{2}\right\rangle,
$$

where

$$
\begin{aligned}
\langle R\rangle & =\frac{1}{N} \sum_{i=1}^{N} R_{i}, \\
\left\langle(R-\langle R\rangle)^{2}\right\rangle & =\frac{1}{N} \sum_{i=1}^{N}\left(R_{i}-\langle R\rangle\right)^{2}, \\
\left\langle\left(1-\frac{S_{\text {com }}}{S}\right)^{2}\right\rangle & =\frac{1}{N} \sum_{i=1}^{N}\left(1-\frac{S_{\text {com } i}}{S_{i}}\right)^{2}, \\
S_{i} & =\pi R_{i}^{2} .
\end{aligned}
$$

Here $i$ is the number index of an individual erythrocyte in the blood smear, $N$ is the total number of erythrocytes in the smear, $S_{i}$ is the area of the $i$ th erythrocyte, $R_{i}$ is the radius of the $i$ th erythrocyte, $\langle R\rangle$ is the mean erythrocyte radius, and $S_{\text {comi }}$ is the maximal area of the common part of the erythrocyte and an equal-sized circle. The parameter $\sigma^{2}$, as determined by (34), can be considered a measure of inhomogeneity of the RBC sample by size and shape. As it can be seen from (30), the parameter $\sigma$ has a simple relationship to the visibility $v$ of the diffraction pattern produced by laser beam scattering on a blood smear. Thus, our analysis demonstrates that inhomogeneity of RBC size and shape in a blood specimen can be assessed experimentally using laser diffraction analysis.

\section{NUMERICAL SIMULATION}

In this section, we will perform numerical modeling of the diffraction pattern produced by laser beam scattering on an inhomogeneous RBC ensemble and find the relationship between the pattern visibility and the parameter describing inhomogeneity of the RBC ensemble, $v=v(\sigma)$.

Let us assume that the parameters $\varepsilon_{1}$ and $\varepsilon_{2}$ of our theoretical model are discrete random variables with identical characteristics. Namely,

$$
\begin{array}{cl}
\varepsilon_{1 i}=\Delta \varepsilon_{i}, & p_{i}=\frac{1}{N}, \quad-\frac{N}{2} \leq i \leq \frac{N}{2}, \\
\varepsilon_{2 j}=\Delta \varepsilon_{j}, & p_{j}=\frac{1}{N}, \quad-\frac{N}{2} \leq j \leq \frac{N}{2} .
\end{array}
$$


The means and dispersions of these values are given by the formulas

$$
\begin{gathered}
\left\langle\varepsilon_{1}\right\rangle=\left\langle\varepsilon_{2}\right\rangle=\sum_{i} \varepsilon_{1 i} p_{i}=\frac{1}{N} \sum_{i=-N / 2}^{N / 2} \varepsilon_{1 i}=\frac{\Delta \varepsilon}{N} \sum_{i=-N / 2}^{N / 2} i=0, \\
\sigma_{1}^{2}=\sigma_{2}^{2}=\left\langle\varepsilon_{1}^{2}\right\rangle=\sum_{i} \varepsilon_{1 i}^{2} p_{i}=\frac{1}{N} \sum_{i=-N / 2}^{N / 2} \varepsilon_{1 i}^{2} \\
=(\Delta \varepsilon)^{2} \frac{1}{N} \sum_{i=-N / 2}^{N / 2} i^{2} .
\end{gathered}
$$

For numerical calculations, we assume $N=10$. Then

$$
\frac{1}{N} \sum_{i=-N / 2}^{N / 2} i^{2}=\frac{2}{N} \sum_{i=1}^{N / 2} i^{2}=\frac{1}{5} \sum_{i=1}^{5} i^{2}=11,
$$

$\sigma_{1}^{2}=11(\Delta \varepsilon)^{2}$ and $\Delta \varepsilon=\sigma_{1} / \sqrt{11}$. Now we make use of $\sigma^{2}=\sigma_{1}^{2}+\frac{1}{2} \sigma_{2}^{2}$. Assuming that $\sigma_{1}^{2}=\sigma_{2}^{2}$, we obtain $\sigma_{1}=\sigma \sqrt{2 / 3}$ and $\Delta \varepsilon=\sigma \sqrt{\frac{2}{33}}$. Thus, the values of $\varepsilon_{1}$ and $\varepsilon_{2}$, and, as a consequence, the characteristics of the RBC ensemble, are fully determined. Based on these data, we can solve the direct problem of diffraction and find the visibility of the diffraction pattern.

Let us introduce the function

$$
G(x)=\left[\frac{2 J_{1}(x)}{x}\right]^{2} .
$$

The distribution of light intensity in the diffraction pattern can be found using the formula

$f(u, v)=\frac{I}{I(0)}=\langle G(q)\rangle=\frac{1}{N^{2} M} \sum_{i=-N / 2}^{N / 2} \sum_{j=-N / 2}^{N / 2} \sum_{k=1}^{M} G\left(q_{i j k}\right)$,

where

$$
q_{i j k}=\left(1+\varepsilon_{1 j}\right) \sqrt{\left(1+\varepsilon_{i}\right)^{2}\left(u \cos \varphi_{k}+v \sin \varphi_{k}\right)^{2}+\left(1-\varepsilon_{i}\right)^{2}\left(-u \sin \varphi_{k}+v \cos \varphi_{k}\right)^{2}}
$$

and

$$
\varphi_{k}=\Delta \varphi k, \quad \Delta \varphi=\frac{2 \pi}{M}, \quad 1 \leq k \leq M .
$$

These formulas were derived using (10). The normalized coordinates $u$ and $v$ are given by

$$
u=\frac{k R_{0}}{z} x, \quad v=\frac{k R_{0}}{z} y .
$$

The angle $\varphi$ determines the orientation of the ellipse representing an erythrocyte in the blood smear. We assume that this angle is a discrete random variable with a uniform distribution function. Thus, we simulate the situation where cell orientations in the blood smear are completely random. Notice that the distribution of light intensity in the diffraction pattern exhibits circular symmetry. Therefore, it can be represented by the distribution of light intensity on an individual transversal ray, i.e., a line going from the center of the pattern to its periphery. In particular, this ray can be directed along the horizontal axis on the observation screen. Then we obtain the function

$$
f(u)=\frac{1}{N^{2} M} \sum_{i=-N / 2}^{N / 2} \sum_{j=-N / 2}^{N / 2} \sum_{k=1}^{M} G\left(q_{i j k}\right),
$$

where

$$
\times \frac{q_{i j k}=\left(1+\varepsilon_{1 j}\right)}{\times \sqrt{\left(1+\varepsilon_{i}\right)^{2}\left(u \cos \varphi_{k}\right)^{2}+\left(1-\varepsilon_{i}\right)^{2}\left(-u \sin \varphi_{k}\right)^{2}} .}
$$

In the numerical calculations, we assumed $N=10$ and $M=100$. This means that, for each value of $\sigma$, the diffraction pattern was calculated for an ensemble of
$N^{2} M=10^{4}$ erythrocytes. In each calculation, we obtain one value of diffraction pattern visibility and thus a single point of the relationship $v=v(\sigma)$. It should be noted that the accuracy of visibility measurement depends on the resolution of the diffraction pattern. In our calculations, the resolution was assumed to be $10^{6}$ pixels. This resolution provides sufficiently high accuracy and can be achieved in practice using standard registering equipment. By repeating the calculations for other values of $\sigma$, we constructed the entire curve $v=V(\sigma)$. This relationship is shown in

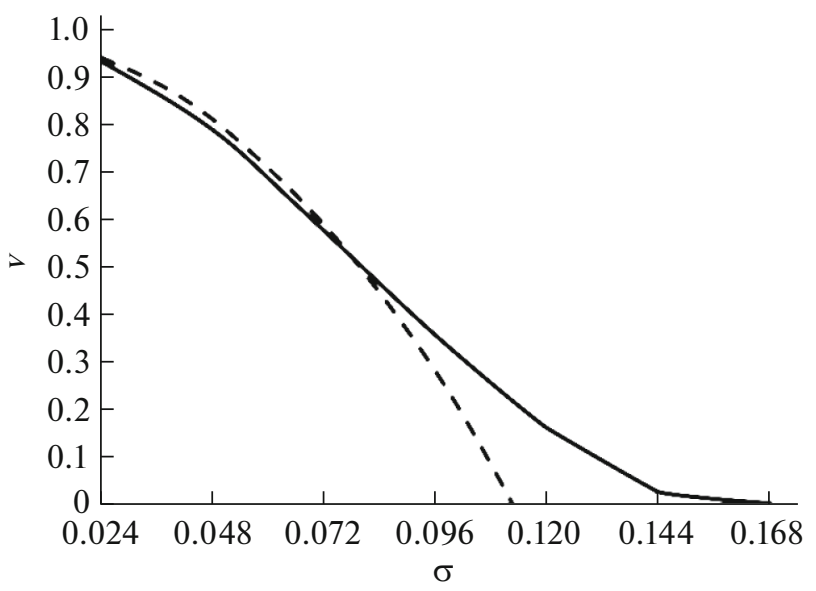

Fig. 2. Relationship between the diffraction pattern visibility and inhomogeneity of an RBC ensemble. The solid curve was obtained by numerical simulation (see text). The dashed curve was obtained using formula (28). 

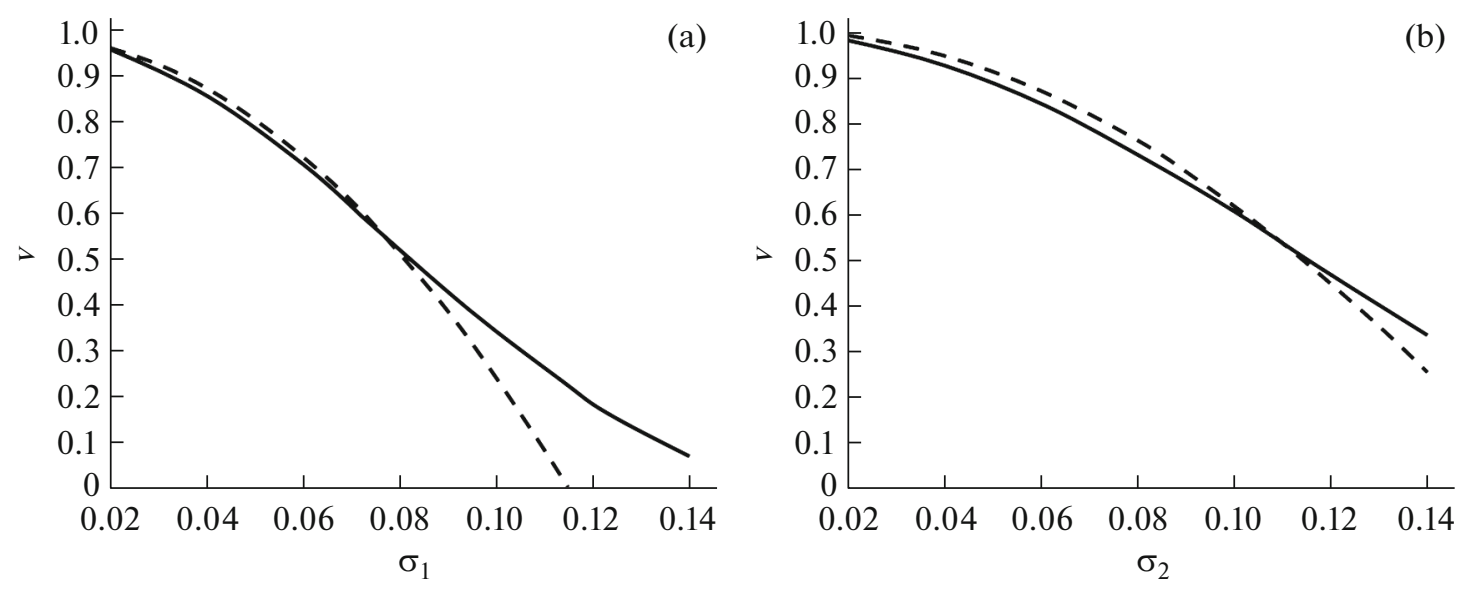

Fig. 3. Relationship between the diffraction pattern visibility and inhomogeneity of an RBC ensemble. The solid curve in panel a was constructed considering only the particle size variation $\sigma_{1}$. The solid curve in panel $b$ was constructed considering only the particle shape variation $\sigma_{2}$. The dashed curves were constructed using formula (28) under assumption that $\sigma_{2}=0$ (a) or $\sigma_{1}=0$ (b).

Fig. 2. For comparison, the dashed curve in the same figure was constructed using formula (28). It can be seen that, for weakly inhomogeneous RBC ensembles, this formula provides a good approximation of the dependence between visibility of the diffraction pattern and the parameter describing inhomogeneity of the RBC ensemble.

In addition, we specifically considered the cases where an RBC ensemble is inhomogeneous only in cell size or only in cell shape. The results of these calculations are shown in Fig. 3. It can be seen that estimate (28) of diffraction pattern visibility shown by the dashed curve exhibits good agreement with the results of numerical simulation in the range of relatively small values of $\sigma_{1}$ and $\sigma_{2}$, i.e., for weakly inhomogeneous $\mathrm{RBC}$ ensembles.

The relationship between the diffraction pattern visibility and inhomogeneity of an RBC ensemble in both size and shape is shown in Fig. 4. In this chart,

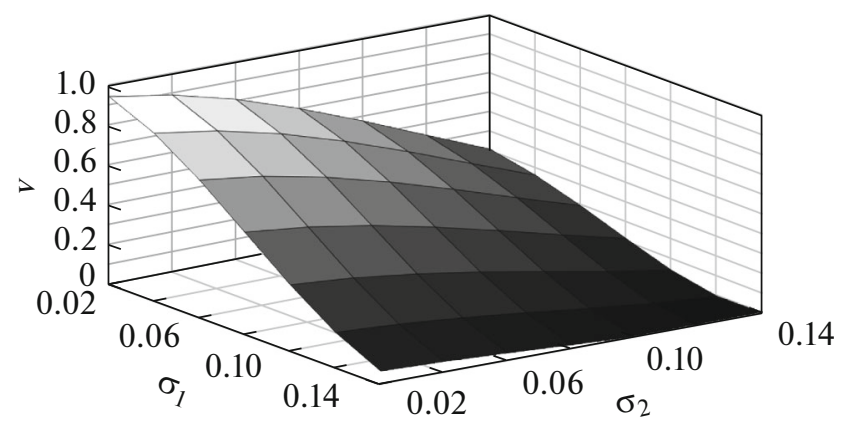

Fig. 4. Visibility of the diffraction pattern as a function of parameters describing inhomogeneity of an RBC ensemble in size $\left(\sigma_{1}\right)$ and shape $\left(\sigma_{2}\right)$. visibility is presented as a surface in three-dimensional space, and the horizontal Cartesian axes represent the values of $\sigma_{1}$ and $\sigma_{2}$.

The numerical data corresponding to Fig. 4 are also presented in Table 1. Dashes signify that the dark ring is so fuzzy because of strong variation in RBC size and shape that there is no local minimum of intensity in the dark ring zone and therefore the notion of diffraction pattern visibility has no sense.

\section{CONCLUSIONS}

The study describes an analytical model of laser beam scattering on an inhomogeneous RBC ensemble. The model takes into account $\mathrm{RBC}$ variation in size, shape, and spatial orientation. A relationship has been established between the visibility of the diffraction pattern as an experimentally determined parameter and a characteristic that serves as a measure of RBC size and shape inhomogeneity in a blood specimen. If RBC variation in size is measured independently (e.g., using a Coulter counter), laser diffraction analysis can be used to assess inhomogeneity of cell shape in a given blood smear. These measurements can be applied in diagnostics of conditions associated with anomalies in the shape of blood cells.

In the present work, we discuss RBC size distribution meaning the distribution by diameter. In the model of flat discs that we utilize here, the distribution by thickness does not have a significant effect on the diffraction pattern but only affects its brightness. The character of the diffraction pattern is affected by the relief of the erythrocyte surface. However, further studies are required to investigate this aspect. 
Table 1. Visibility of the diffraction pattern as a function of parameters describing RBC variation in size $\left(\sigma_{1}\right)$ and shape $\left(\sigma_{2}\right)$

\begin{tabular}{c|c|c|c|c|c|c|c}
\hline \multirow{2}{*}{$\sigma$} & \multicolumn{7}{|c}{$\sigma_{1}$} \\
\cline { 2 - 8 } & 0.02 & 0.04 & 0.06 & 0.08 & 0.10 & 0.12 & 0.14 \\
\hline 0.14 & 0.3187 & 0.2715 & 0.2016 & 0.1208 & 0.0458 & 0.0000 & - \\
0.12 & 0.4492 & 0.3901 & 0.3021 & 0.1994 & 0.0995 & 0.0227 & - \\
0.10 & 0.5817 & 0.5110 & 0.4054 & 0.2814 & 0.1589 & 0.0581 & 0.0000 \\
0.08 & 0.7049 & 0.6238 & 0.5025 & 0.3597 & 0.2171 & 0.0967 & 0.0180 \\
0.06 & 0.8118 & 0.7220 & 0.5873 & 0.4281 & 0.2684 & 0.1319 & 0.0379 \\
0.04 & 0.8942 & 0.7977 & 0.6529 & 0.4814 & 0.3087 & 0.1601 & 0.0551 \\
0.02 & 0.9464 & 0.8456 & 0.6942 & 0.5150 & 0.3344 & 0.1783 & 0.0666 \\
\hline
\end{tabular}

SUPPLEMENT

Let us consider an ellipse with the semiaxes $a$ and $b$ and an equal-sized circle with the radius $\sqrt{a b}$. The center of the ellipse coincides with the center of the circle. The point of intersection between the circle and the ellipse in the quadrant $x>0, y>0$ will be characterized using Cartesian coordinates $x, y$, as well as polar coordinates $r, \varphi$. Then

$$
\begin{gathered}
\frac{x^{2}}{a^{2}}+\frac{y^{2}}{b^{2}}=1, \\
x^{2}+y^{2}=a b, \\
\tan \varphi=\frac{y}{x}, \\
x=r \cos \varphi, \quad y=r \sin \varphi .
\end{gathered}
$$

Let us introduce an auxiliary angle $\psi$ given by the formulas

$$
\sin \psi=\frac{y}{b}, \quad \cos \phi=\frac{x}{a} .
$$

The relationship between the polar angle $\varphi$ and the auxiliary angle $\psi$ is as follows:

$$
\frac{y}{x}=\tan \varphi=\frac{b}{a} \tan \psi
$$

The ellipse equation in the polar coordinates is

$$
r(\varphi)=\frac{a b}{\sqrt{a^{2} \sin ^{2} \varphi+b^{2} \cos ^{2} \varphi}} .
$$

Let us calculate the area of an ellipse sector. Using polar coordinates, we obtain:

$$
\begin{aligned}
& S_{\mathrm{el}}=\int_{0}^{\varphi} d \varphi \int_{0}^{r(\varphi)} r d r=\frac{1}{2} \int_{0}^{\varphi} r^{2}(\varphi) d \varphi \\
& =\frac{1}{2} a^{2} b^{2} \int_{0}^{\varphi} \frac{d \varphi}{a^{2} \sin ^{2} \varphi+b^{2} \cos ^{2} \varphi} .
\end{aligned}
$$

To calculate this integral, we will use the variable $\psi$.
We take into account the following formulas

$$
\begin{aligned}
& \sin ^{2} \varphi=\frac{\tan ^{2} \varphi}{1+\tan ^{2} \varphi}=\frac{\frac{b^{2}}{a^{2}} \tan ^{2} \psi}{1+\frac{b^{2}}{a^{2}} \tan ^{2} \psi} \\
& =\frac{b^{2} \sin ^{2} \psi}{a^{2} \cos ^{2} \psi+b^{2} \sin ^{2} \psi}, \\
& \cos ^{2} \varphi=\frac{1}{1+\tan ^{2} \varphi}=\frac{1}{1+\frac{b^{2}}{a^{2}} \tan ^{2} \psi} \\
& =\frac{a^{2} \cos ^{2} \psi}{a^{2} \cos ^{2} \psi+b^{2} \sin ^{2} \psi}, \\
& a^{2} \sin ^{2} \varphi+b^{2} \cos ^{2} \varphi=\frac{a^{2} b^{2}}{a^{2} \cos ^{2} \psi+b^{2} \sin ^{2} \psi}, \\
& d \tan \varphi=\frac{d \varphi}{\cos ^{2} \varphi}=\frac{b}{a} d \tan \psi=\frac{b}{a} \frac{d \psi}{\cos ^{2} \psi}, \\
& d \varphi=\frac{b}{a} \frac{\cos ^{2} \varphi}{\cos ^{2} \psi} d \psi=\frac{b}{a} \frac{a^{2} \cos ^{2} \psi}{a^{2} \cos ^{2} \psi+b^{2} \sin ^{2} \psi} \frac{d \psi}{\cos ^{2} \psi} \\
& =\frac{a b}{a^{2} \cos ^{2} \psi+b^{2} \sin ^{2} \psi} d \psi, \\
& \frac{d \varphi}{a^{2} \sin ^{2} \varphi+b^{2} \cos ^{2} \varphi}=\frac{a^{2} \cos ^{2} \psi+b^{2} \sin ^{2} \psi}{a^{2} b^{2}} \\
& \times \frac{a b}{a^{2} \cos ^{2} \psi+b^{2} \sin ^{2} \psi} d \psi=\frac{d \psi}{a b} .
\end{aligned}
$$

Hence

$S_{\mathrm{el}}=\frac{1}{2} a^{2} b^{2} \int_{0}^{\varphi} \frac{d \varphi}{a^{2} \sin ^{2} \varphi+b^{2} \cos ^{2} \varphi}=\frac{1}{2} a b \int_{0}^{\psi} d \psi=\frac{1}{2} a b \psi$.

Thus, the area of a sector of an ellipse with the semiaxes $a$ and $b$ is given by

$$
S_{\mathrm{el}}=\frac{1}{2} a b \psi .
$$

Here, the relationship between the angle $\psi$ and the polar angle $\varphi$ is given by (S1). A sector of the circle with the radius $\sqrt{a b}$ corresponding to the polar angle $\varphi$ has the following area:

$$
S_{\text {circ }}=\frac{1}{2} a b \varphi .
$$

Next, let us find the coordinates of the point of intersection of the ellipse and th equations of an ellipse and a circle:

$$
\frac{x^{2}}{a^{2}}+\frac{y^{2}}{b^{2}}=1, \quad x^{2}+y^{2}=a b,
$$




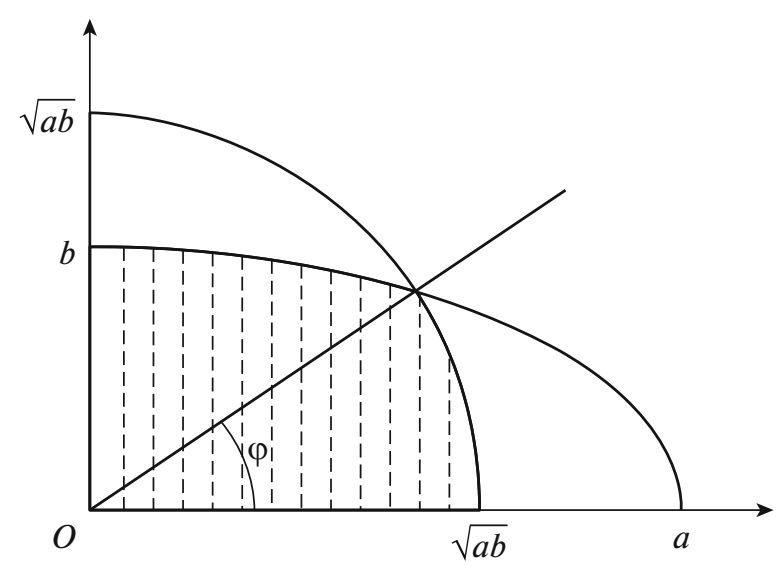

Fig. 5. Sectors of a circle and an equal-sized ellipse. The common part of the ellipse and the circle is shaded.

and solve them for $x$ and $y$. We obtain

$$
x=a \sqrt{\frac{b}{a+b}}, \quad y=b \sqrt{\frac{a}{a+b}} .
$$

These formulas determine the Cartesian coordinates of the point of intersection of the ellipse and the circle of equal area. The angular coordinate $\varphi$ of this point is given by

$$
\tan \varphi=\frac{y}{x}
$$

Hence

$$
\tan \varphi=\frac{y}{x}=\frac{b \sqrt{a}}{a \sqrt{b}}=\sqrt{\frac{b}{a}} .
$$

At the same time

$$
\tan \psi=\frac{a}{b} \tan \varphi=\sqrt{\frac{a}{b}}
$$

Let us calculate the parameter $M$ of ellipse asymmetry as defined by (31). Due to symmetry, it suffices to consider the sector of the pattern lying in the quadrant $x>0$ and $y>0$. For the sake of definiteness, let us assume $a>b$. The outlines of an ellipse and a circle for this case are shown in Fig. 5.

In Fig. 5, the common part of the circle and the ellipse is shaded. The open parts of the ellipse and the circle have the same area $\Delta S$, which represents the measure of ellipse asymmetry. Obviously, this area can be found as the difference between the areas of the ellipse and circle sectors corresponding to the same polar angle $\varphi$, that is:

$$
\Delta S=S_{\text {el }}-S_{\text {circ }} .
$$

Using formulas (S2) and (S3), we obtain

$$
\Delta S=\frac{1}{2} a b(\psi-\varphi)
$$

A quarter of the circle has the area $S=\frac{1}{4} \pi a b$. Hence

$$
M=\frac{\Delta S}{S}=\frac{2}{\pi}(\psi-\varphi) .
$$

Thus, we obtain

$$
M=\frac{2}{\pi}(\psi-\varphi),
$$

where

$$
\tan \varphi=\sqrt{\frac{b}{a}}, \quad \tan \psi=\sqrt{\frac{a}{b}} .
$$

This is true for an ellipse with arbitrary eccentricity.

Now let us consider a specific case of an ellipse with small eccentricity. We need to find out how the parameter $M$, which can be measured for a real erythrocyte, is related to the parameter $\varepsilon_{2}$ employed in our theoretical model and determined by formulas (7). Assuming that $\left|\varepsilon_{2}\right| \ll 1$, we obtain

$$
\tan \varphi=\sqrt{\frac{b}{a}}=\sqrt{\frac{1-\varepsilon_{2}}{1+\varepsilon_{2}}} \approx \sqrt{\left(1-\varepsilon_{2}\right)^{2}}=1-\varepsilon_{2}
$$

and

$$
\tan \psi=\sqrt{\frac{a}{b}}=\sqrt{\frac{1+\varepsilon_{2}}{1-\varepsilon_{2}}} \approx \sqrt{\left(1+\varepsilon_{2}\right)^{2}}=1+\varepsilon_{2} .
$$

Therefore, the angles $\varphi$ and $\psi$ are close to $45^{\circ}$. Let us present them in the following form:

$$
\varphi=\frac{\pi}{4}-\alpha, \quad \psi=\frac{\pi}{4}+\beta,
$$

where $\alpha, \beta \ll 1$. Then

$$
\tan \varphi=1-2 \alpha, \quad \tan \psi=1+2 \beta
$$

From (S7), (S8), and (S10), it follows that

$$
\alpha=\frac{\varepsilon_{2}}{2}, \quad \beta=\frac{\varepsilon_{2}}{2} .
$$

Using (S9) and (S11), we find

$$
\psi-\varphi=\alpha+\beta=\varepsilon_{2} .
$$

By substituting this expression into formula (S5), we obtain $M=\frac{2}{\pi} \varepsilon_{2}$, hence

$$
\varepsilon_{2}=\frac{\pi}{2} M
$$

\section{ACKNOWLEDGMENTS}

The authors are grateful to A.V. Priezzhev and A.E. Lugovtsov for valuable discussion of the results.

\section{FUNDING}

This work was supported by the Russian Foundation for Basic Research, project no. 17-29-03507. 


\section{CONFLICT OF INTEREST}

The authors declare that they have no conflicts of interest.

\section{REFERENCES}

1. Yu. S. Yurchuk, V. D. Ustinov, S. Yu. Nikitin, and A. V. Priezzhev, Quantum Electron. 46, 515 (2016). https://doi.org/10.1070/QEL16108

2. S. Yu. Nikitin, A. E. Lugovtsov, A. V. Priezzhev, and V. D. Ustinov, Quantum Electron. 41, 843 (2011). https://doi.org/10.1070/QE2011v041n09ABEH014675

3. K. V. Patel, L. Ferrucci, W. B. Ershler, D. L. Longo, and J. M. Guralnik, Arch. Intern. Med. 169, 515 (2009).

4. C. P. Titcomb, On the Risk 33, 30 (2017).

5. B. H. Foy, J. C. T. Carlson, E. Reinertsen, R. P. I. Valls, R. Pallares Lopez, E. Palanques-Tost, C. Mow, M. B. Westover, A. D. Aguirre, and J. M. Higgins, JAMA Netw Open 3, e2022058 (2020). https://doi.org/10.1001/jamanetworkopen.2020.22058

6. K. S. Shifrin, Introduction to Ocean Optics (Gidrometeoizdat, Leningrad, 1983) [in Russian].

7. R. Xu, Particle Characterization: Light Scattering Methods (Springer, 2002). https://doi.org/10.1007/0-306-47124-8

8. G. I. Kozinets, V. M. Pogorelov, D. A. Shmarov, S. F. Boev, and V. V. Sazonov, Blood Cells-Modern Technologies for their Analysis (Triada-Farm, Moscow, 2002) [in Russian].

9. V. N. Lopatin, A. V. Priezzhev, I. V. Aponasenko, A. D. Shepelevich, N. V. Lopatin, V. V. Pozhilenkova, and P. V. Prostakova, Light Scattering Methods in the Analysis of Dispersed Biological Media (Fizmatlit, Moscow, 2004) [in Russian].

10. Y. Yang, Z. Zhang, X. Yang, J. H. Yeo, L. Jiang, and D. Jiang, J. Biomed. Opt. 9, 995 (2004). https://doi.org/10.1117/1.1782572

11. V. D. Ustinov, Math. Models Comput. Simul. 9, 561 (2017). https://doi.org/10.1134/S2070048217050131

12. S. Yu. Nikitin, A. V. Priezzhev, A. E. Lugovtsov, V. D. Ustinov, and A. V. Razgulin, J. Quant. Spectrosc. Radiat. Transfer 146, 365 (2014). https://doi.org/10.1016/j.jqsrt.2014.05.012
13. V. D. Ustinov and E. G. Tsybrov, Inverse Probl. Sci. Eng. 28, 1633 (2020). https://doi.org/10.1080/17415977.2020.1761801

14. M. A. Yurkin and A. G. Hoekstra, J. Quant. Spectrosc. Radiat. Transfer 112, 2234 (2011). https://doi.org/10.1016/j.jqsrt.2011.01.031

15. M. A. Yurkin, A. G. Hoekstra, R. S. Brock, and J. Q. Lu, Opt. Express 15, 17902 (2007). https://doi.org/10.1364/OE.15.017902

16. Y. Eremin, E. Eremina, and T. Wriedt, Opt. Commun. 244, 15 (2005). https://doi.org/10.1016/j.optcom.2004.09.037

17. T. Wriedt, J. Hellmers, E. Eremina, and R. Schuh, J. Quant. Spectrosc. Radiat. Transfer 100, 444 (2006). https://doi.org/10.1016/j.jqsrt.2005.11.057

18. https://www.malvernpanalytical.com/ru/products/ product-range/mastersizer-range.

19. A. N. Tikhonov and V. B. Glasko, USSR Comput. Math. Math. Phys. 4, 236 (1964). https://doi.org/10.1016/0041-5553(64)90254-X

20. A. N. Tikhonov, A. V. Goncharsky, V. V. Stepanov, and A. G. Yagola, Numerical Methods for the Solution of IllPosed Problems (Springer, Berlin, 2013). https://doi.org/10.1007/978-94-015-8480-7

21. J. B. Riley and Y. C. Agrawal, Appl. Opt. 30, 4800 (1991). https://doi.org/10.1364/AO.30.004800

22. S. Yu. Nikitin, A. E. Lugovtsov, and A. V. Priezzhev, Quantum Electron. 40, 1074 (2010). https://doi.org/10.1070/QE2010v040n12ABEH01450 4

23. S. A. Akhmanov and S. Yu. Nikitin, Physical Optics (Nauka, Moscow, 2004; Oxford Univ. Press, Oxford, 1997).

24. G. P. Sarkisyan, A. G. Sarkisyan, and A. M. Danielyan, Izv. NAN Armen., Fiz. 54, 293 (2019).

25. G. P. Sarkisyan, Biol. Zh. Armen. 72 (1-2), 102 (2020).

26. G. P. Sarkisyan, Dokl. NAN Armen. 120, 45 (2020).

27. E. Janke, F. Emde, and F. Lösch, Tables of Higher Functions (Teubner, Stuttgart, 1960; McGraw-Hill, New York, 1960).

Translated by D. Timchenko 